Check for updates

Cite this: RSC Adv., 2019, 9, 33345

Received 6th September 2019 Accepted 1st October 2019

DOI: $10.1039 / c 9 r a 07155 e$

rsc.li/rsc-advances

\section{Enhanced removal of fluoride by zirconium modified tea waste with extrusion treatment: kinetics and mechanism $\dagger$}

\author{
Liping Mei, D † Chuanyi Peng, \$ Huanhuan Qiao, Fei Ke, Jin Liang, Ruyan Hou, \\ Xiaochun Wan* and Huimei Cai*
}

To improve the adsorption efficiency of tea-based biosorbents for removing fluoride in drinking water, the novel and effective adsorbent was formed by treating tea waste with extrusion technology. In this study, the extrusion technology was applied to the preparation of adsorbents for the first time. A low-priced and more efficient adsorbent was prepared by loading zirconium onto extruded tea waste (EXT-Zr). Extruded tea waste increased the surface pore size, which could provide more loading sites for zirconium. The EXT-Zr effectively removed fluoride from water in a $\mathrm{pH}$ range of 3.0-10.0, which is wider than the $\mathrm{pH}$ range of zirconium-loaded tea waste (Tea-Zr). The adsorption was fitted by a pseudo-second order kinetic model and the Langmuir adsorption model. The maximum adsorption capacity was $20.56 \mathrm{mg} \mathrm{g}^{-1}$. The EXT-Zr adsorbent was characterized by Scanning electron microscopy (SEM), Energy-Dispersive Spectroscopy (EDS), X-ray diffraction (XRD), a Brunauer-Emmett-Teller (BET) method, Fourier-transform infrared (FTIR) and X-ray photoelectron spectroscopy (XPS) to prove the mechanism of how EXT-Zr adsorbs fluoride. The results proved that EXT-based adsorbent will be effective for the enhanced removal of fluoride in drinking water.

\section{Introduction}

Fluoride is a widespread element found mainly in nature. ${ }^{1}$ Fluoride is considered as an essential trace element for human beings ${ }^{2}$ and can prevent dental caries by reducing the demineralization rate of enamel or by increasing the rate of remineralization. ${ }^{3}$ Although fluoride is beneficial, excess fluoride can lead to multifarious diseases, such as arthritis, skeletal fluorosis, infertility, osteoporosis and so on. ${ }^{4,5}$ Due to the hazards of excessive fluoride, the World Health Organization (WHO) has stipulated a limit of fluoride in drinking water $\left(1.5 \mathrm{mg} \mathrm{L}^{-1}\right){ }^{6}$

Fluoride pollution of drinking water has been recognized as a public health risk in most of the world. ${ }^{7}$ Fluoride in water is caused by pollution with industrial wastewater and by geochemical weathering of rocks, for example, fluorapatite, which is the main source of fluoride toxicity. Groundwater resources in China, India and some western countries (such as the United States and Mexico) contain high concentrations of

School of Tea \& Food Science and Technology, Anhui Agricultural University, State Key Laboratory of Tea Plant Biology and Utilization, No. 130 Changjiangxi Road, Hefei 230036, Anhui, People's Republic of China.E-mail: hml20@sina.com;xcwan@ahau. edu.cn

† Electronic supplementary information (ESI) available. See DOI: 10.1039/c9ra07155e

\$ Liping Mei and Chuanyi Peng contributed equally to this work. fluoride. ${ }^{8}$ Therefore, it is significant to decrease the fluoride content in water prepared for drinking by the public.

There are a few methods to remove fluoride, including coagulation/precipitation methods, ${ }^{9}$ ion-exchange processes, ${ }^{\mathbf{1 0}}$ membrane processes, ${ }^{7}$ and adsorption processes. ${ }^{11}$ Each technique has advantages and limitations, including the conditions under which it performs the best. Adsorption technology is widely used, because of its safety, simplicity, inexpensive, and high efficiency. ${ }^{12}$ The adsorbent materials most generally utilized for defluoridation include activated alumina, ${ }^{\mathbf{1 3}}$ activated carbon, ${ }^{8}$ bone char ${ }^{14}$ and zeolites. ${ }^{15}$ More and more people use biopolymers as adsorbent materials because of their nontoxicity, good biocompatibility, and low cost. Biopolymer materials are usually modified with metals (iron, aluminum, etc.) such as pectin, ${ }^{16}$ alginate, ${ }^{17}$ cellulose,${ }^{18}$ chitosan, ${ }^{19}$ etc. However, these absorbents have several disadvantages, such as difficulty in handling and a narrow $\mathrm{pH}$ range for effective adsorption. ${ }^{20}$ Therefore, it is meaningful to synthesize an effective and low-priced adsorbent for fluoride removal.

To improve the adsorption performance of biological materials, many physical or chemical methods have been adopted, such as ball milling, ${ }^{21}$ enzymatic hydrolysis, ${ }^{22}$ and composite metal modification. ${ }^{20}$ Extrusion technology is a thermal mechanical preprocessing method for materials, due to its low cost, controllable temperature and screw speed, high shear and excellent processing ability. Twin-screw extrusion is the main method at present. Compared with ball mill and homogenizer, 
twin-screw extruder has higher solid phase load and lower energy consumption. ${ }^{23}$ Twin-screw extrusion is a thermomechanical process, and the chemical and thermal properties of the extruded products are similar to those of the original biological materials because no compounds are removed. ${ }^{24}$ Twin-screw extrusion technology may change the size of lignocellulose by increasing the surface area of lignocellulose, creating more pores and voids. ${ }^{25}$ At present, twin-screw extrusion technology has been used to pretreat many kinds of lignocellulosic materials such as straw, ${ }^{26}$ pineapple leaf, ${ }^{27}$ bagasse $^{28}$ and corn stover. ${ }^{29}$ However, twin-screw extrusion technology has not been applied to biosorbents so far.

In this study, a new adsorbent (EXT-Zr) was prepared by combining twin-screw extrusion of tea byproducts with the zirconium. The adsorption kinetics and adsorption isotherms were calculated, and the adsorbent dosage, solution $\mathrm{pH}$, contact time, initial fluoride concentration and co-existing ions were varied in the starting reaction. The mechanism by which the adsorbent removed fluoride is proposed.

\section{Materials and methods}

\subsection{Experimental materials}

Spent tea leaves were gathered from a tea factory. Tea was washed with boiling, distilled water several times, until the washing liquid was colorless. The washed materials dried in a drying oven at $80^{\circ} \mathrm{C}$ for $8 \mathrm{~h}$ and crushed, and were processed by an extruder (DSE32, twin-screw extruder, Jinan Shengrun Machinery Co., Ltd). After adjusting the moisture content of the materials to $30 \%$, the extrusion was carried out, feeding speed was $12 \mathrm{~Hz}$, and the temperature of the barrel zone 1 was $60{ }^{\circ} \mathrm{C}$, the temperature of the barrel zone 2 was $135{ }^{\circ} \mathrm{C}$, the temperature of the barrel zone 3 was $140{ }^{\circ} \mathrm{C}$, and the screw rotation speed was $35 \mathrm{~Hz}$. After that, extruded tea waste (EXT) were obtained. The Tea and EXT were ground and sifted mesh 60. The Tea and EXT obtained were used in the following experiment to prepare composite biosorbents.

Chemical reagents used in this experiment were analytical grade (A.R.) and procured from Sinopharm Chemical Reagent Co., Ltd. (Shanghai, China).

\subsection{Preparation of biosorbent composites}

A $0.4 \mathrm{~mol} \mathrm{~L}^{-1} \mathrm{Zr}$ solution was made by mixing $\mathrm{ZrOCl}_{2} \cdot 8 \mathrm{H}_{2} \mathrm{O}$ in distilled water. Tea $(5 \mathrm{~g})$ or EXT $(5 \mathrm{~g})$ was immersed in $100 \mathrm{~mL}$ of the $\mathrm{Zr}$ solution at $300 \mathrm{rpm}$ at $60^{\circ} \mathrm{C}$ for $1 \mathrm{~h}$ on a heated magnetic stirrer. Then, $2.0 \mathrm{~mol} \mathrm{~L}^{-1} \mathrm{NaOH}$ was added dropwise to regulate the mixture $\mathrm{pH}$ to 7.0 , kept stirring for $30 \mathrm{~min}$. Finally, the mixture was centrifuged, washed a few times with distilled water, then dried in a drying oven at $70{ }^{\circ} \mathrm{C}$ for $10 \mathrm{~h}$.

\subsection{Biosorbents characterization}

Microscopic surface morphology and characteristics were detected by scanning electron microscopy (SEM, Sirion from FEI). Surface elements of the adsorbent were detected by Energy Dispersive Spectrometer (EDS, Sirion from FEI). The crystalline structure of the adsorbent was detected by X-ray diffraction
(XRD, X'Pert PRO from PHILIPS). The chemical bonding environments and adsorption sites were analyzed by X-ray photoelectron spectroscopy (XPS, Thermo Scientific Instrument Co. USA) Surface functional groups were detected by FourierTransform Infrared Spectroscopy (FTIR, Thermo Scientific Instrument Co. USA). The pore size distribution and surface area were detected by $\mathrm{N}_{2}$ chemisorptions measurements with Brunauer-Emmett-Teller (BET) analysis.

\subsection{Batch adsorption experiment}

Batch adsorption experiments were executed to determine the adsorption of fluoride on the adsorbents. The impacts of adsorbent dosage (0.5-2.0 $\left.\mathrm{g} \mathrm{L}^{-1}\right)$, solution $\mathrm{pH}$ (3-12), contact time (1-300 $\mathrm{min}$ ), initial fluoride concentration (5-400 $\mathrm{mg} \mathrm{L}^{-1}$ ), and other co-existing anions $\left(\mathrm{SO}_{4}{ }^{2-}, \mathrm{NO}_{3}{ }^{-}, \mathrm{Cl}^{-}\right.$, and $\left.\mathrm{HCO}_{3}{ }^{-}\right)$ were examined. The initial solution $\mathrm{pH}$ was regulated with $\mathrm{HCl}$ and/or $\mathrm{NaOH}$. Batch adsorption works were performed with $25 \mathrm{~mL}$ of fluoride solution in $50 \mathrm{~mL}$ centrifuge tubes at room temperature $\left(25^{\circ} \mathrm{C}\right)$. After the addition of the test dose of biosorbents into the fluoride solutions, the centrifuge tube was shaken at $300 \mathrm{rpm}$ for 90 minutes on the shaker. After the above steps, the mixed solution was divided by filtration and the residual fluoride ion concentration was detected by ion selective electrode method. ${ }^{21}$

The removal efficiency $(\eta)$ for the fluoride ions and the total fluoride adsorbed $\left(\mathrm{mg} \mathrm{g}^{-1}\right)$ at equilibrium $\left(q_{\mathrm{e}}\right)$ were obtained from eqn (1) and (2).

$$
\begin{gathered}
\eta=\frac{C_{0}-C_{1}}{C_{0}} \times 100 \% \\
q_{\mathrm{e}}=\frac{V\left(C_{0}-C_{1}\right)}{m}
\end{gathered}
$$

where $\eta(\%)$ represents the removal efficiency of the fluoride, $C_{0}$ represents the initial fluoride concentrations $\left(\mathrm{mg} \mathrm{L}^{-1}\right)$ and $C_{1}$ represents the after-adsorption fluoride concentrations $\left(\mathrm{mg} \mathrm{L}{ }^{-1}\right), m$ represents the dry weight of the adsorbent $(\mathrm{g})$, and $V$ represents the volume of fluoride solution $(\mathrm{mL})$. Each work was performed three times, and adsorption capacity was showed as the average \pm standard deviation.

\section{Result and discussion}

\subsection{Tea waste versus extruded tea waste}

The surface topography of the Tea and EXT were observed by SEM. The surface of the Tea was smooth (Fig. 1a). The surface of the EXT was rough and had tiny pores (Fig. 1b and c). The surface areas of the Tea and EXT were 2.085 and $3.126 \mathrm{~m}^{2} \mathrm{~g}^{-1}$, respectively. The pore sizes were measured by BET, and the average pore radius was $0.44 \mathrm{~nm}$ in the Tea and $14.42 \mathrm{~nm}$ in the EXT (Fig. 2a). The pore diameter in the EXT much larger than in the Tea, and this increase is attributable to the extrusion process. These changes may increase the surface area and thus the adsorption capacity.

The defluoridation of Tea, EXT, Tea-Zr and EXT-Zr were compared in experiments using $0.030 \mathrm{~g}$ adsorbent and $25 \mathrm{~mL}$ $10 \mathrm{mg} \mathrm{\textrm {L } ^ { - 1 }}$ fluoride solution (Fig. 2b). Both the Tea and EXT 

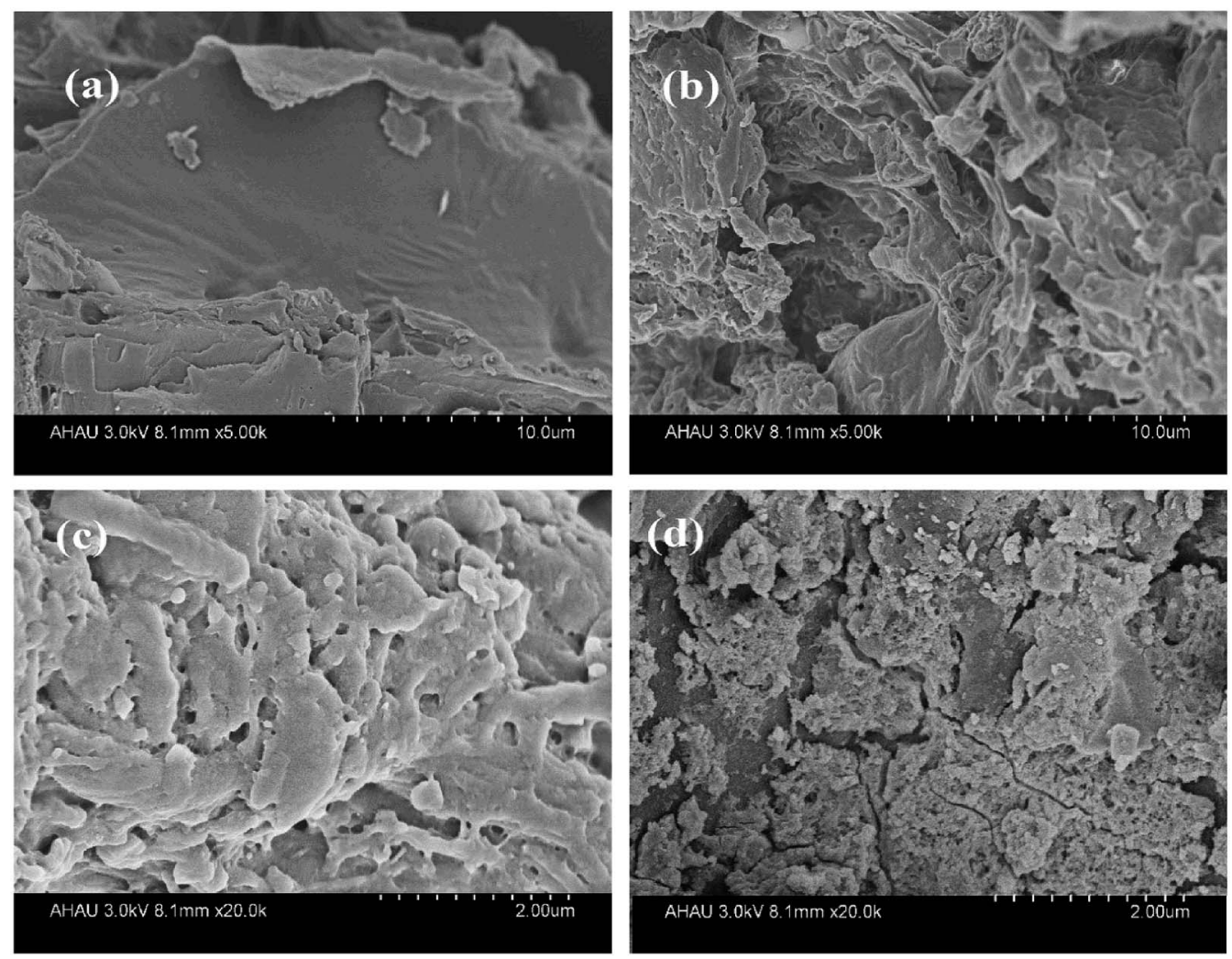

Fig. 1 SEM images of tea waste (a), extruded tea waste (EXT, b), at higher magnification (EXT, c) and extruded tea waste with zirconium (EXT-Zr, d).

adsorbents had extremely low fluoride adsorption efficiencies. The adsorption rate of Tea-Zr was $61 \%$. The adsorption rate of EXT-Zr, using the extruded tea as the scaffold, reached $97 \%$. Since the extrusion process increased the pore size, exposing a greater number of functional groups, the EXT was able to load more zirconium than the unextruded tea, thereby greatly improving the adsorption efficiency of fluoride.

\subsection{Batch defluoridation experiment}

3.2.1. Effect of adsorption dosage. The effect of the adsorbent dosage (for Tea-Zr and EXT-Zr) on fluoride removal is displayed in Fig. 3a. The adsorption doses ranged from 0.32 to $2 \mathrm{~g} \mathrm{~L}^{-1}$ while the other parameters remained constant, at a concentration of $10 \mathrm{mg} \mathrm{L}^{-1}$ fluoride in a solution of $\mathrm{pH}$ of 6.8 \pm 0.3 and a contact time of $90 \mathrm{~min}$. Increasing the EXT-Zr dose from 0.32 to $1.2 \mathrm{~g} \mathrm{~L}^{-1}$ rised the adsorption rate from $26 \%$ up to $97 \%$. Perhaps this is because the increased dose of EXT-Zr will provide more active sites. After the amount of EXT-Zr adsorbent exceeded $1.2 \mathrm{~g} \mathrm{~L}^{-1}$, the fluoride removal rate does not significantly increase, indicating that the reduce in the concentration of fluoride in the solution at these levels of adsorbent are so low that fluoride adsorption slows as fewer fluoride ions are available. ${ }^{30}$ On the other hand, the sorbent made with unexpanded
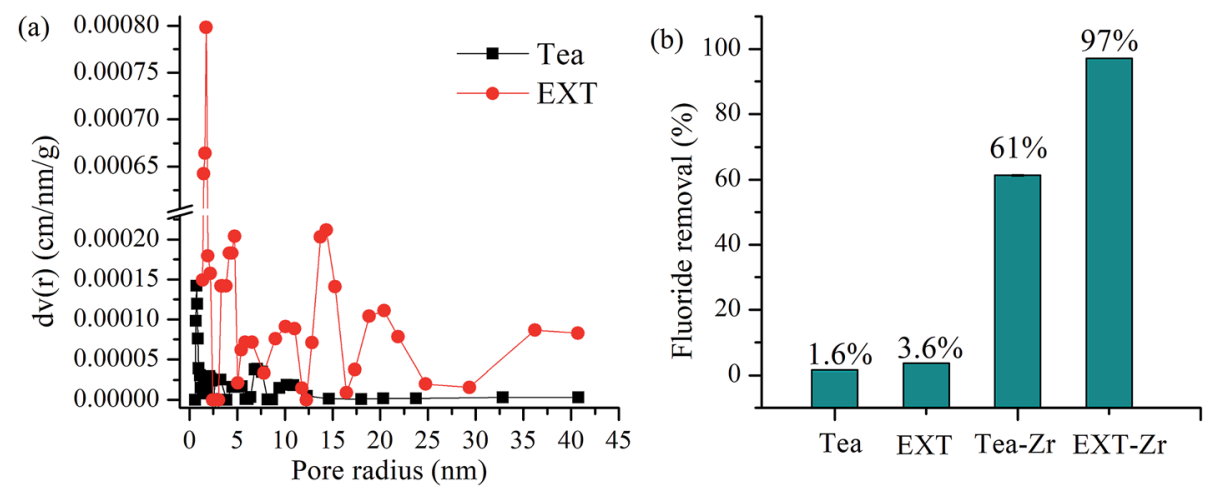

Fig. 2 Pore size distribution in Tea and EXT (a), comparison of the fluoride removal efficiencies of Tea, EXT, Tea-Zr, and EXT-Zr (b). 

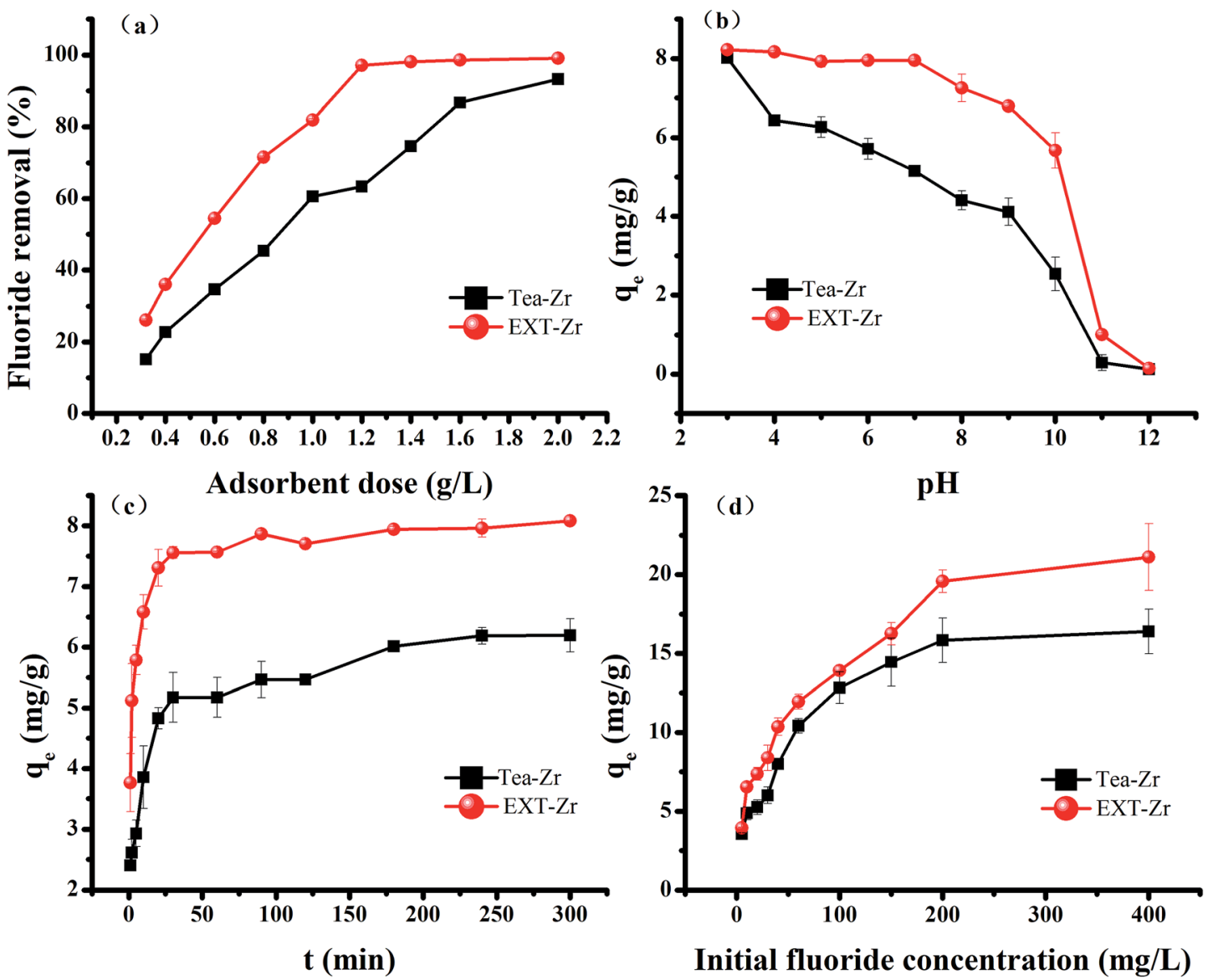

Fig. 3 The effect of adsorbent dose (a), initial pH (b), contact time (c) and initial fluoride concentration (d) on the fluoride adsorption capacity of the tea waste loaded with zirconium (Tea-Zr) and extruded tea loaded with zirconium (EXT-Zr).

tea, Tea-Zr, did not reach equilibrium at $1.2 \mathrm{~g} \mathrm{~L}^{-1}$, at which point the defluoridation rate was only $61 \%$. The adsorption rate continued to increase with the dose of Tea-Zr. These experimental results showed that EXT-Zr had a better overall adsorption effect. For further experiments, a biosorbent dose was $1.2 \mathrm{~g} \mathrm{~L}^{-1}$ was used to balance cost and effectiveness and to reflect the capacity of the modified tea-based biosorbent.

3.2.2. Effect of solution $\mathbf{p H}$. The effect of $\mathrm{pH}$ on fluoride adsorption is displayed in Fig. 3b. The adsorption experiment was carried out for $90 \mathrm{~min}$ at a concentration of $10 \mathrm{mg} \mathrm{L}^{-1}$ fluoride, $1.2 \mathrm{~g} \mathrm{~L}^{-1}$ biosorbent and at a pH of 3 to 12 . Compared with Tea-Zr, EXT-Zr had a higher adsorption capacity for fluoride across all $\mathrm{pH}$ values. In extremely acidic systems, the adsorption capacity was higher because the surface of the biosorbent was positively charged and the electrostatic adsorption increases the adsorption capacity. At a $\mathrm{pH}$ value of 5 , the amount of fluoride adsorbed decreased slightly. As the $\mathrm{pH}$ value was between 3 to 7 , the EXT-Zr maintained a high adsorption capacity $\left(q_{\mathrm{e}}\right)$, at around $8 \mathrm{mg} \mathrm{\textrm {g } ^ { - 1 }}$. The adsorption capacity of EXT-Zr decreases slightly at $\mathrm{pH}$ values of 8-10. Most biosorbents

Table 1 The pseudo-first order and pseudo-second order kinetic modeling results for adsorption of fluoride by $\mathrm{Tea}^{-\mathrm{Zr}}$ and $\mathrm{EXT}-\mathrm{Zr}{ }^{a}$

\begin{tabular}{|c|c|c|c|c|}
\hline Models & Parameters & Description & Tea-Zr & EXT-Zr \\
\hline $\begin{array}{l}\text { The pseudo-first order model } \\
\qquad \log \left(q_{\mathrm{e}}-q_{t}\right)=\log q_{\mathrm{e}}-\frac{K_{1}}{2.303}\end{array}$ & $\begin{array}{l}K_{1}\left(\min ^{-1}\right) \\
q_{\mathrm{e}}\left(\mathrm{mg} \mathrm{g}^{-1}\right) \\
R^{2}\end{array}$ & $\begin{array}{l}\text { The rate constant of pseudo-first order model } \\
\text { The equilibrium adsorption capacity } \\
\text { Correlation coefficient }\end{array}$ & $\begin{array}{l}0.0215 \\
3.96 \\
0.8042\end{array}$ & $\begin{array}{l}0.0155 \\
1.84 \\
0.8555\end{array}$ \\
\hline The pseudo-second order model $\frac{t}{q_{t}}=\frac{1}{K_{2} q_{\mathrm{e}}^{2}}+\frac{t}{q_{\mathrm{e}}}$ & $\begin{array}{l}K_{2}\left(\mathrm{~g} \mathrm{mg}^{-1} \min ^{-1}\right) \\
q_{\mathrm{e}}\left(\mathrm{mg} \mathrm{g}^{-1}\right) \\
R^{2}\end{array}$ & $\begin{array}{l}\text { The rate constant of pseudo-first order model } \\
\text { The equilibrium adsorption capacity } \\
\text { Correlation coefficient }\end{array}$ & $\begin{array}{l}0.0235 \\
6.22 \\
0.9977\end{array}$ & $\begin{array}{l}0.0516 \\
8.07 \\
0.9998\end{array}$ \\
\hline
\end{tabular}

${ }^{a} q_{t}=$ the amount of fluoride ion adsorbed at time; $t=$ time (min). 
Table 2 Langmuir and Freundlich isotherm constants for adsorption of fluoride by Tea-Zr and EXT-Zr biosorbents ${ }^{a}$

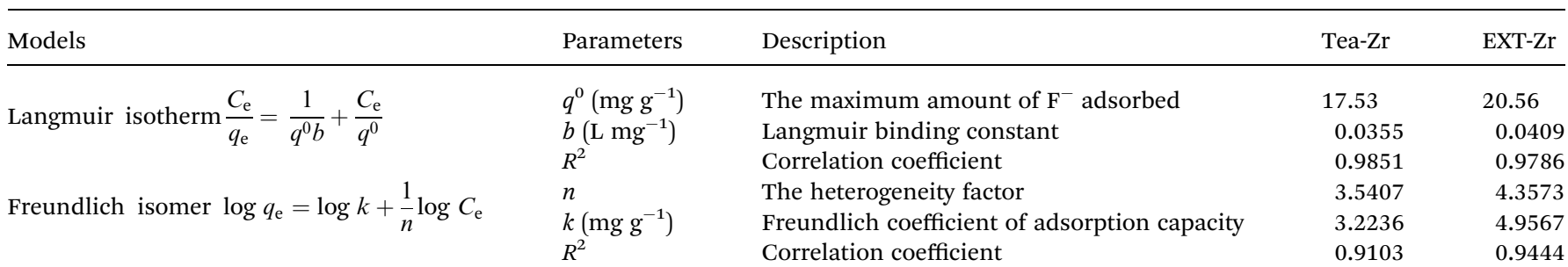

${ }^{a} C_{\mathrm{e}}=$ the solution concentration at equilibrium; $q_{\mathrm{e}}=$ adsorption capacity at equilibrium.

remove fluoride by anion exchange between $\mathrm{OH}^{-}$and $\mathrm{F}^{-} .{ }^{31}$ The adsorption capacity of Tea-Zr decreases to greater extents than does that of EXT-Zr as the $\mathrm{pH}$ of the solution increases. Adsorbents effectively removed fluoride in the $\mathrm{pH}$ range of 3-10. This showed that EXT-Zr better adsorbs fluoride across a wider pH range. However, the adsorption capacity of EXT-Zr was extremely low at a $\mathrm{pH}$ value of 11-12. With the increase of $\mathrm{pH}$, $\mathrm{OH}^{-}$may be competing with $\mathrm{F}^{-}$for $\mathrm{Zr}$ binding sites, grabbing adsorption sites on the surface of the biosorbent, resulting in a decrease in the ability to adsorb $\mathrm{F}^{-} .^{16}$

3.2.3. Effect of contact time. The effect of contact time on fluoride adsorption is displayed in Fig. 3c. The adsorption experiment was carried out at a $\mathrm{pH}$ of $6.8 \pm 0.3$ and a concentration of $10 \mathrm{mg} \mathrm{L}^{-1}$ of fluoride solution for a contact time of 1 to $300 \mathrm{~min}$. The results clearly showed that the adsorption capacity of the biosorbent increases as the reaction time increases. The relationship between the adsorption of fluoride ion and the contact time of Tea-Zr was similar to that of EXT-Zr. However, the adsorption effect of EXT-Zr was much better than that of Tea-Zr. Tea-Zr never absorbed as much fluoride even after $300 \mathrm{~min}$. EXT-Zr removed more than $90 \%$ of the fluoride in the first $30 \mathrm{~min}$, indicating that the biosorbent has enough sites to adsorb fluoride in the early stage of the reaction. ${ }^{32}$ The adsorption reached equilibrium at $90 \mathrm{~min}$, thus, $90 \mathrm{~min}$ was chosen as the reaction time for the next works.

3.2.4. Effect of initial fluoride concentration. The effect of initial concentration on fluoride adsorption is shown in Fig. 3d. The adsorption experiment was performed with an initial fluoride concentration ranging from 5 to $400 \mathrm{mg} \mathrm{L}^{-1}$ at $\mathrm{pH} 6.8 \pm 0.3$ and for $90 \mathrm{~min}$. With the initial fluoride concentration increased, the removal of fluoride capacity of the adsorbent increased. The initial fluoride concentration reached adsorption equilibrium at $200 \mathrm{mg} \mathrm{L}^{-1}$. Adsorption first increased and then reached an equilibrium. This may be caused by the relatively large quantity of active sites within the biosorbent at a low fluoride concentration ${ }^{32}$ that become saturated as the initial concentration increases, and the surface active sites gradually reach saturation, so that the adsorption gradually moves towards stability. ${ }^{33}$

\subsection{Adsorption kinetics}

To explore the theory of fluoride adsorption by zirconium-loaded extruded tea waste, the experimental data was simulated by kinetic models ${ }^{34}$ (Table 1, Fig. S1a and $\mathrm{b} \dagger$ ). The values of $K_{1}, K_{2}$ and $q_{\mathrm{e}}$ were calculated from the slope and intercept. For EXT-Zr, the $R^{2}$ value of the pseudo-second order model was 0.9998 , larger than the pseudo-first order model $\left(R^{2}\right.$ value of 0.8555$)$. This indicated that the pseudo-second order kinetics model better describes the adsorption action by EXT-Zr. This also demonstrated that the adsorption reaction can be a classified as a chemisorption process. ${ }^{35,36}$

\subsection{Adsorption isotherm}

The adsorption data were analyzed by the Langmuir and Freundlich isotherms models. The Langmuir isotherm presumes that the adsorption occurs in a single-layer on the surface of a homogeneous biosorbent. ${ }^{37}$ Whereas the Freundlich adsorption isotherm presumes that the adsorption is

Table 3 Maximum adsorption value of EXT-Zr and other adsorbent materials on fluoride

\begin{tabular}{|c|c|c|c|c|}
\hline Adsorbent & $q^{0}\left(\mathrm{mg} \mathrm{g}^{-1}\right)$ & $\mathrm{pH}$ & Contact time (min) & Reference \\
\hline AC-Zr & 5.400 & 4 & 180 & 8 \\
\hline UTP-Zr & 12.43 & $3-10$ & 120 & 21 \\
\hline MBC & 9.000 & $2-10$ & 5 & 6 \\
\hline $\mathrm{Fe}(\mathrm{III})$-ASF & 0.400 & 7 & 60 & 47 \\
\hline PA-Fe(III)-ASF & 0.200 & 7 & 60 & 47 \\
\hline Ficus benghalensis leaves biosorbent & 2.200 & 7 & 60 & 50 \\
\hline Cocos nucifera Linn. root biosorbent & 2.000 & 7 & 90 & 51 \\
\hline RSL & 15.80 & 7.94 & & 52 \\
\hline EXT-Zr & 20.56 & $3-10$ & 90 & This study \\
\hline
\end{tabular}


heterogeneous and that the adsorption process is not limited to a single layer, but is a multiphase adsorption process. ${ }^{38}$

The $R^{2}$ value (0.9786) of the Langmuir isotherm model for the adsorbent EXT-Zr was higher than that of the Freundlich model (0.9444) (Fig. S1c, $\mathrm{d} \dagger$ and Table 2). Similarly, the $R^{2}$ value $(0.9851)$ of the Langmuir isotherm model for Tea-Zr was larger than that of the Freundlich model (0.9103). Therefore, the Langmuir model showed a good consistency in predicting the defluoridation by the two adsorbents. The modeling also indicated that the surface properties of the EXT-Zr adsorbent are uniform. The calculated maximum removal fluoride capacity of EXT-Zr was $20.56 \mathrm{mg} \mathrm{g}^{-1}$. Compared with other biosorbent in recent years (Table 3), extruded tea waste has stronger adsorption capacity and can be used as a good biological adsorption material.

\subsection{Effect of co-existing ions}

The effects of co-existing anions on fluoride adsorption is shown in Fig. S2. $\dagger$ These ions, such as $\mathrm{Cl}^{-}, \mathrm{SO}_{4}{ }^{2-}, \mathrm{NO}_{3}{ }^{-}$, or $\mathrm{HCO}_{3}{ }^{-}$, exist in both wastewater and groundwater, and these ions would be present and probably competing with fluoride in time of the adsorption process. The experimental results displayed that the $\mathrm{Cl}^{-}$and $\mathrm{NO}_{3}{ }^{-}$have insufficient effect on the adsorbent, and the fluoride removal effect was hardly affected, even at $200 \mathrm{mg} \mathrm{L}{ }^{-1}$. The $\mathrm{SO}_{4}{ }^{2-}$ slightly affected the defluoridation process, resulting in a slight decrease in fluoride removal efficiency. The $\mathrm{HCO}_{3}{ }^{-}$had a greater impact on the adsorbent, decreasing the fluoride adsorption by EXT-Zr by nearly $50 \%$ at the low concentration of $50 \mathrm{mg} \mathrm{L}^{-1}$. This interference may be due to the initial increase in $\mathrm{pH}$ caused the significant decrease in fluoride adsorption. ${ }^{20}$

\subsection{Characterization of the samples and study of the adsorption mechanism}

3.6.1. Characterization of the biosorbent samples. The elemental make-up of the EXT-Zr before and after (EXT-Zr-F) adsorption of fluoride were characterized by EDS (Fig. S3 $\dagger$ ), respectively. EDS analysis showed the elemental peaks, with only carbon and oxygen in the EXT (Fig. S3a†). But in EXT-Zr, there was an additional peak, for zirconium, which illustrated that the zirconium was successfully loaded onto the EXT (Fig. S2b $\dagger$ ). After fluoride adsorption, another peak appeared for fluoride, which illustrated that the fluoride was successfully adsorbed onto EXT-Zr (Fig. S3c $\dagger$ ).

The surface of the EXT-Zr appeared to be very rough in SEM images (Fig. 1d). XRD analysis was performed to further study the structure of Tea and EXT-Zr (Fig. S3d $\dagger$ ). In the EXT-Zr sample, there was not a significant aqueous zirconium peak, indicating that the amorphous nature of the EXT adequately supported the zirconium ions and that ions were not freely floating in the solution. Amorphous materials are known to have more active sites on their surfaces, such that they can be used as adsorbents. ${ }^{33}$

3.6.2. FTIR study. In order to better understand the adsorption process of fluoride, it was further analyzed by FTIR (Fig. 4). In the raw tea waste, the peak at $3423.36 \mathrm{~cm}^{-1}$ is attributed to the hydrogen bonding of -OH stretching, while the peak of the strong stretching vibration at $1632.63 \mathrm{~cm}^{-1}$ and $1437.78 \mathrm{~cm}^{-1}$ is attributed to the presence of carboxyl groups. ${ }^{39}$ After extrusion, the hydroxyl group was shifted to $3146.98 \mathrm{~cm}^{-1}$, the peak of the carboxyl group was shifted to $1651.80 \mathrm{~cm}^{-1}$ and $1451 \mathrm{~cm}^{-1}$, probably due to the effect of water and high temperature during the extrusion process. After loading zirconium, the carboxyl groups shifted to $1636.44 \mathrm{~cm}^{-1}$ and $1437.92 \mathrm{~cm}^{-1}$, indicating that zirconium interacts with the carboxyl group. ${ }^{40}$ A new peak appears at $433.92 \mathrm{~cm}^{-1}$ for $\mathrm{Zr}-\mathrm{O},{ }^{41}$ and a new peak at $1546.15 \mathrm{~cm}^{-1}$ represents $\mathrm{Zr}-\mathrm{OH}{ }^{42}$ After the fluoride adsorption, the peak of $\mathrm{Zr}-\mathrm{OH}$ became weak, indicating that the process of removing the fluoride involved the hydroxyl group of the metal oxide.

3.6.3. XPS study. To explore the possible adsorption mechanism underlying fluoride removal, XPS measurements were made, as shown in Fig. 5 and Table S1. $\dagger$ The zirconium peak appeared clearly in the loaded EXT (Fig. 5a and b). The elemental ratio of Zr(Iv) in EXT and EXT-Zr were 0 and $6.02 \%$, respectively, indicating that zirconium was successfully loaded

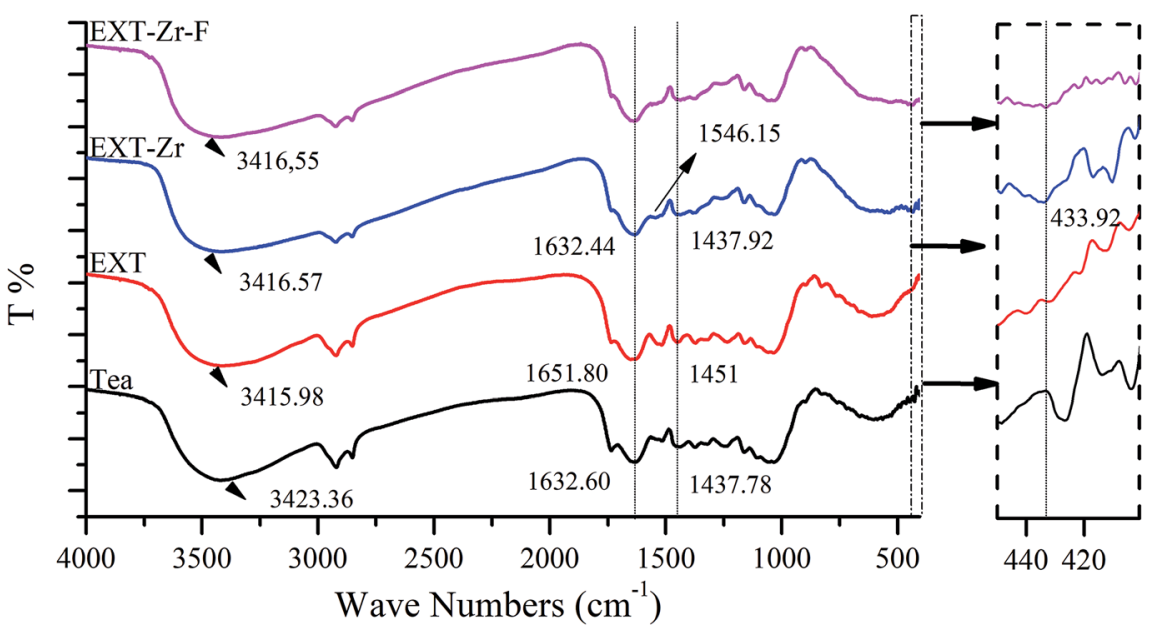

Fig. 4 FTIR spectra of tea, tea waste with zirconium (Tea-Zr), extruded with zirconium before (EXT-Zr) and after (EXT-Zr-F) fluoride adsorption. 
onto the EXT. After fluoride adsorption, a peak of fluoride appeared clearly (Fig. 5c), indicating that fluorine was successfully adsorbed. A narrow XPS spectra (194-172) showed that the peak corresponding to the binding energy of $\mathrm{Zr} 3 \mathrm{~d}$ before adsorption of fluoride was located at $182.56 \mathrm{eV}$ and after fluoride adsorption shifted to $182.65 \mathrm{eV}$ (Fig. 5d), indicating a change in the zirconium species. Binding with the most electronegative element, fluoride, results in a decrease in the electron concentration of $\mathrm{Zr}$, which increases the $3 \mathrm{~d}$ binding energy. At the same time, a peak of fluoride appeared, and the relative content of fluoride on the surface after EXT-Zr adsorption increased to $2 \%$, which showed that fluoride was adsorbed by the adsorbent successfully. In addition, after adsorption the binding energy of the $\mathrm{F}$ 1s peak $(684.8 \mathrm{eV})$ was higher than that

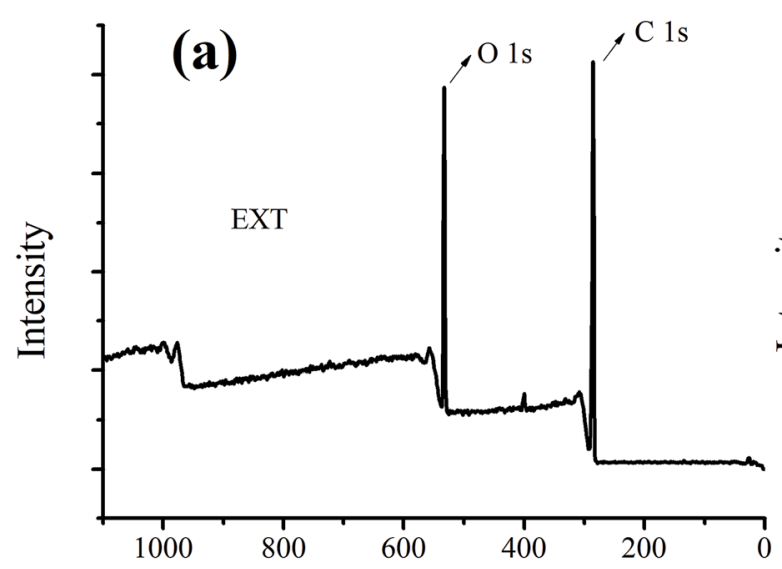

Binding Energy (ev)
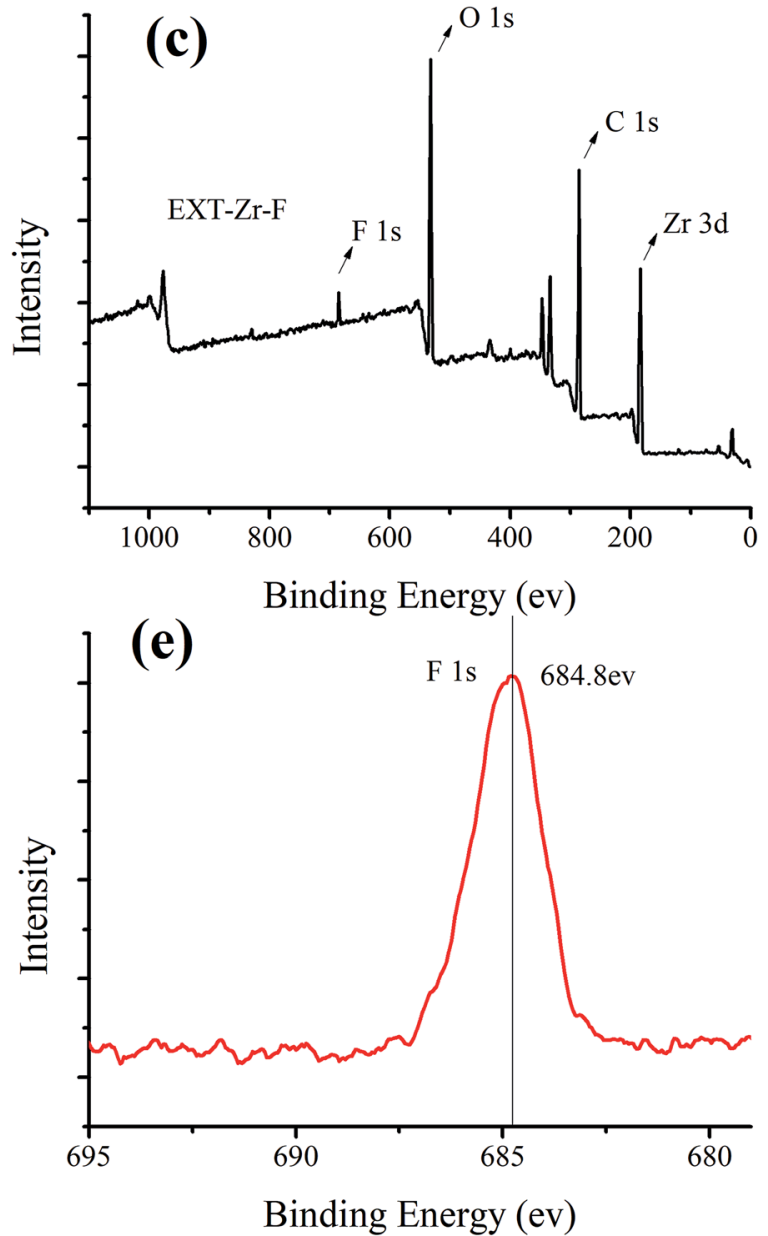

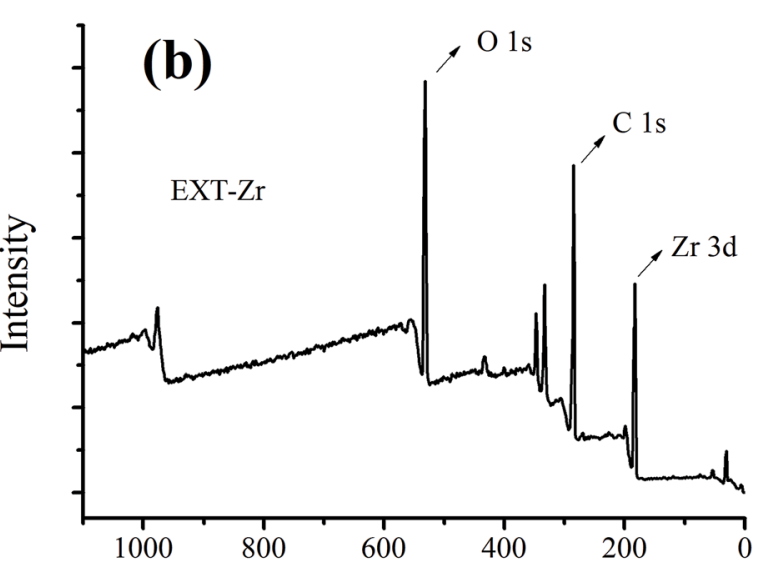

Binding Energy (ev)

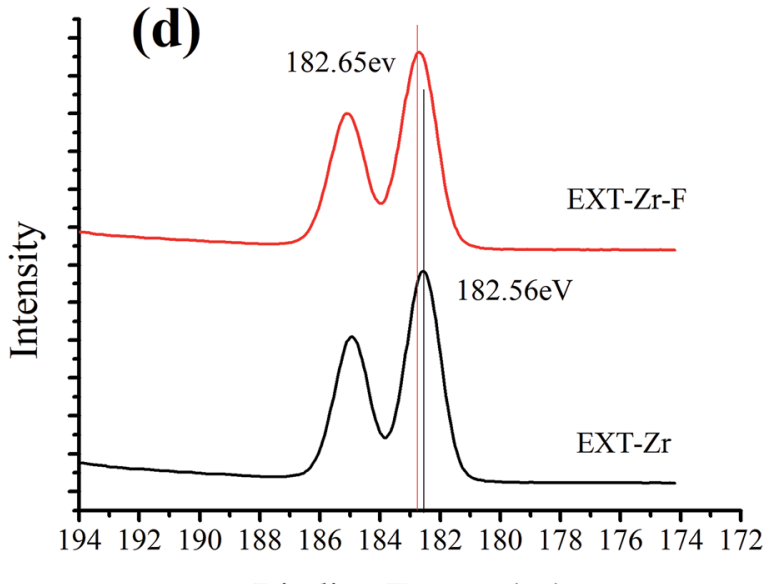

Binding Energy (ev) spectra, (d) Zr 3d and (e) F 1s. 


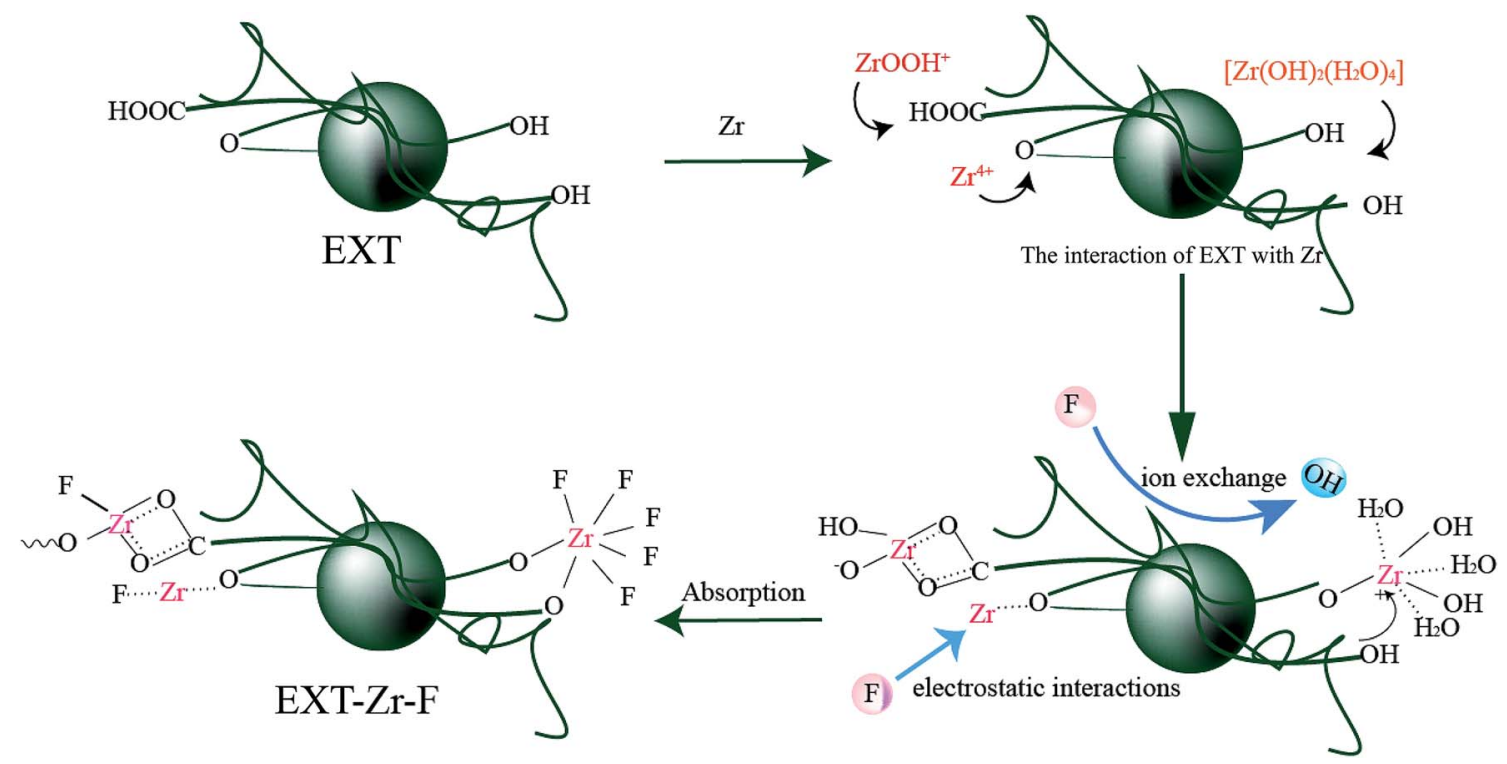

Fig. 6 The proposed mechanism of fluoride adsorption by extruded tea loaded with zirconium (EXT-Zr).

of $\mathrm{NaF}$ (684.5 eV) (Fig. 5e), demonstrating that EXT-Zr adsorbed fluoride mainly through chemical adsorption. ${ }^{43}$ These results indicated that the fluoride removal process directly involved the $\mathrm{Zr}$ and confirmed that the strong interaction between $\mathrm{Zr}$ and $\mathrm{F}$ had high electronegativity. ${ }^{44}$

The analysis of the extruded tea product and how it adsorbs fluoride allowed for the proposal of the following model. EXT-Zr can form a complex with water through dissociation between the charged surfaces. The adsorption process is mainly through ion exchange, with some electrostatic interactions (Fig. 6).

In a mildly acidic or neutral system the number of sites carrying positive charges on the biosorbent is reduced, some even become negatively charged, resulting in the reduction of the protonation of the hydroxyl. Under these conditions, ion exchange between the hydroxide ion and the fluoride ion mainly occurs. ${ }^{45}$

In an extremely acidic system, the hydroxyl group is protonated and the electrostatic interaction becomes one of the adsorption methods. The surface of the EXT-Zr will be positively charged, which electrostatically attracts the negatively charged $\mathrm{F}^{-}$, thus enhancing fluoride reduction. ${ }^{21}$

\section{Conclusion}

A novel biosorbent was made by loading zirconium with twinscrew extrusion treatment. Through the extrusion process, the pore size of the tea waste increased to expose more functional groups. Therefore, EXT-Zr had a better adsorption effect than Tea-Zr. EXT-Zr also defluoridated the aqueous solution over a wide $\mathrm{pH}$ range. The kinetics model of the adsorption indicates that the process was via chemical absorption. The Langmuir isotherm model presented the better fit with high $R^{2}$ values and figured out a maximum adsorption capacity of $20.56 \mathrm{mg} \mathrm{g}^{-1}$. These results indicated that the modification of tea waste through extrusion can improve the adsorption efficiency of a zirconium-loaded biosorbent.

\section{Conflicts of interest}

There are no conflicts to declare.

\section{Acknowledgements}

This work was financially supported by The National Natural Science Foundation of China (No. 31770730), the Changjiang Scholars and Innovative Research Team in University (No. IRT1101) and the Earmarked Fund for Modern Agro-industry Technology Research System in Tea Industry (No. CARS-19, the Ministry of Agriculture of P. R. China).

\section{References}

1 S. Tangsir, L. D. Hafshejani, A. Lähde, M. Maljanen, A. Hooshmand, A. A. Naseri, H. Moazed, J. Jokiniemi and A. Bhatnagar, Chem. Eng. J., 2016, 288, 198-206.

2 G. Singh, B. Kumari, G. Sinam, N. Kumar and S. Mallick, Environ. Pollut., 2018, 239, 95-108.

3 S. Aubrey and W. P. T. James, Public Health Nutr., 2014, 17, 2176-2184.

4 H. J. Gao, Y. Q. Jin and J. L. Wei, Environ. Monit. Assess., 2013, 185, 3687-3695.

5 X. Fan, D. J. Parker and M. D. Smith, Water Res., 2003, 37, 4929-4937.

6 N. B. Dewage, A. S. Liyanage, C. U. Pittman Jr, D. Mohan and T. Mlsna, Bioresour. Technol., 2018, 263, 258.

7 K. K. Yadav, N. Gupta, V. Kumar, S. A. Khan and A. Kumar, Environ. Int., 2018, 111, 80-108.

8 A. Mullick and S. Neogi, Ultrason. Sonochem., 2018, 45, 65. 
9 S. Dubey, M. Agarwal and A. B. Gupta, J. Mol. Liq., 2018, 266, 349-360.

10 S. Mukherjee and G. N. Halder, Environ. Prog. Sustainable Energy, 2016, 35, 1305-1316.

11 D. H. Phillips, B. Sen Gupta, S. Mukhopadhyay and A. K. Sen Gupta, J. Environ. Manage., 2018, 215, 132-142.

12 V. E. dos Anjos, J. R. Rohwedder, S. Cadore, G. Abate and M. T. Grassi, Appl. Clay Sci., 2014, 99, 289-296.

13 G. Patra, P. Das, S. Chakraborty and B. C. Meikap, J. Environ. Sci. Health, Part A: Toxic/Hazard. Subst. Environ. Eng., 2018, 53, 1-8.

14 N. M. Zúñigamuro, A. Bonillapetriciolet, D. I. Mendozacastillo, H. E. Reynelávila and J. C. Tapiapicazo, J. Fluorine Chem., 2017, 197, 63-73.

15 M. Dessalegne, F. Zewge and I. Diaz, J. Chem. Technol. Biotechnol., 2017, 92, 595-603.

16 S. Raghav and D. Kumar, Carbohydr. Polym., 2019, 203, 430440.

17 S. Raghav, S. Nehra and D. Kumar, J. Mol. Liq., 2019, 284, 203-214.

18 S. Raghav, S. Nehra and D. Kumar, Process Saf. Environ. Prot., 2019, 127, 211-225.

19 H. Hu, L. Yang, Z. Lin, X. Xiang, X. Jiang and L. Hou, Int. J. Biol. Macromol., 2018, 114, 256-262.

20 H. M. Cai, G. J. Chen, C. Y. Peng, Z. Z. Zhang, Y. Y. Dong, G. Z. Shang, X. H. Zhu, H. J. Gao and X. C. Wan, Appl. Surf. Sci., 2015, 328, 34-44.

21 H. Cai, L. Xu, G. Chen, C. Peng, F. Ke, Z. Liu, D. Li, Z. Zhang and X. Wan, Appl. Surf. Sci., 2016, 375, 74-84.

22 I. S. Ng, X. Wu, X. Yang, Y. Xie, Y. Lu and C. Chen, Bioresour. Technol., 2013, 145, 297-301.

23 J. Li, M. Thompson and D. J. W. Lawton, J. Polym. Environ., 2019, 27, 643-651.

24 D. Theng, G. Arbat, M. Delgado-Aguilar, B. Ngo, L. Labonne, P. Mutje and P. Evon, Eur. J. Wood Wood Prod., 2019, 77, 1532.

25 A. Duque, P. Manzanares and M. Ballesteros, Renewable Energy, 2017, 114, 1427-1441.

26 C. H. Choi and K. K. Oh, Bioresour. Technol., 2012, 110, 349354.

27 F. Berzin, T. Amornsakchai, A. Lemaitre, E. Di Giuseppe and B. Vergnes, Polym. Compos., 2018, 39, 4115-4122.

28 A. S. A. da Silva, R. S. Sobral Teixeira, T. Endo, E. P. S. Bon and S.-H. Lee, Green Chem., 2013, 15, 1991-2001.

29 H. Liu, B. Pang, Y. Zhao, J. Lu, Y. Han and H. Wang, Fuel, 2018, 221, 21-27.
30 G. Zhang, Z. He and W. Xu, Chem. Eng. J., 2012, 183, 315-324.

31 P. Liang, R. An, R. Li and D. Wang, Int. J. Biol. Macromol., 2018, 111, 255-263.

32 S. Mukherjee, S. Dutta, S. Ray and G. Halder, Environ. Sci. Pollut. Res., 2018, 1-17.

33 L. Xu, G. Chen, C. Peng, H. Qiao, F. Ke, R. Hou, D. Li, H. Cai and X. Wan, Carbohydr. Polym., 2017, 160, 82-89.

34 K. S. Prasad, Y. Amin and K. Selvaraj, J. Hazard. Mater., 2014, 276, 232-240.

35 Q. Zhou, X. Lin, B. Li and X. Luo, Chem. Eng. J., 2014, 256, 306-315.

36 Z. Rahimi, A. A. Zinatizadeh and S. Zinadini, Environ. Technol., 2018, 1-11.

37 M. Rosa, R. Di Felice and S. Corni, Langmuir, 2018, 34, 14749-14756.

38 Z. Yu, C. Xu, K. Yuan, X. Gan, C. Feng, X. Wang, L. Zhu, G. Zhang and D. Xu, J. Hazard. Mater., 2017, 346, 82-92.

39 H. Cai, G. Chen, C. Peng, L. Xu, Z. Zhang, F. Ke and X. Wan, RSC Adv., 2015, 5, 101819-101825.

40 J. Wang, L. Wu, J. Li, D. Tang and G. Zhang, J. Alloys Compd., 2018, 753, 422-432.

41 L. Halla Velazquez-Jimenez, R. H. Hurt, J. Matos and J. Rene Rangel-Mendez, Environ. Sci. Technol., 2014, 48, 1166-1174.

42 X. Dou, D. Mohan, C. U. Pittman Jr and S. Yang, Chem. Eng. J., 2012, 198, 236-245.

43 L. Wang, Y. Xie, J. Yang, X. Zhu, Q. Hu, X. Li and Z. Liu, RSC Adv., 2017, 7, 54291-54305.

44 Y. Jia, B. S. Zhu, K. S. Zhang, Z. Jin, B. Sun, T. Luo, X. Y. Yu, L. T. Kong and J. H. Liu, Chem. Eng. J., 2015, 268, 325-336.

45 Y. Liu, Q. Fan, S. Wang, Y. Liu, A. Zhou and L. Fan, Chem. Eng. J., 2016, 306, 174-185.

46 Y. Zhang and K. Huang, RSC Adv., 2019, 9, 7767-7776.

47 H. T. Mwakabona, H. R. Mlay, B. Van der Bruggen and K. N. Njau, J. Hazard. Mater., 2019, 362, 99-106.

48 H. Y. H. Chang, Y.-L. Kuo and J. C. Liu, Sci. Total Environ., 2019, 652, 1331-1338.

49 R. Jha, U. Jha, R. K. Dey, S. Mishra and S. K. Swain, Desalin. Water Treat., 2015, 53, 2144-2157.

50 A. M. George and A. R. Tembhurkar, Sustainable Chem. Pharm., 2018, 10, 125-133.

51 A. M. George and A. R. Tembhurkar, Chin. J. Chem. Eng., 2019, 27, 92-99.

52 T. A. M. Msagati, B. B. Mamba, V. Sivasankar and K. Omine, Appl. Surf. Sci., 2014, 301, 235-243. 